\title{
Do rods influence the hue of foveal stimuli?
}

\author{
STEVEN L. BUCK, LAURA P. THOMAS, NICK HILLYER, AND ERIC M. SAMUELSON \\ Department of Psychology University of Washington, Seattle, Washington
}

(Received March 8, 2006; AcCePTEd March 28, 2006)

\begin{abstract}
To understand the generality and mechanisms of previously reported rod hue biases, we examined whether they are present for small foveal stimuli by comparing the wavelengths of the three spectral unique hues under dark-adapted and flash-bleached conditions. Rod green bias (shift of unique yellow) and rod blue bias (shift of unique green) were found for some observers with $1^{\circ}$-diameter foveal stimuli, the size most likely to stimulate rods. Smaller stimuli $\left(0.2^{\circ}\right.$ and $0.6^{\circ}$ diameter), which were least likely to stimulate rods, produced no large or consistent differences between dark-adapted and bleached conditions. This suggests that rod hue biases result from the local stimulation of rods by light, not from remote suppression by dark-adapted, unstimulated rods, and not from bleaching light artifacts.
\end{abstract}

Keywords: Human color vision, Rod-cone interaction, Foveal vision

\section{Introduction}

The balance of hues seen in large, dim, mesopic stimuli can differ between light-adapted and dark-adapted conditions, apparently because of the activity of rod photoreceptors under dark-adapted conditions. We have consistently found three such rod hue biases: red and blue biases at shorter wavelengths and a green bias at longer wavelengths (e.g., Knight \& Buck, 2002, 2003; Buck et al., 2000, 1998; Thomas \& Buck, 2004). These rod hue biases are most prominent for stimuli centered outside the fovea but some observers show some of these for $1^{\circ}$ - to $2^{\circ}$-diameter foveally centered stimuli (Buck et al., 2000).

This study explored whether any of these rod hue biases would be found when the stimuli were very small and confined to the central fovea, for both practical and theoretical reasons. The practical interest is to determine what, if any laboratory or realworld conditions are safe from rod influence and when it is advisable to evaluate or test for the influence of rods either in laboratory or practical situations involving chromatic discrimination or descriptions of color appearance.

The theoretical interest is to choose between two fundamentally different explanations of the substrate of rod hue biases. We have previously advanced a local-additive explanation for rod hue biases: under dark adapted conditions, signals from rod photoreceptors evoked by the test light selectively add or combine with cone signals that are also evoked by the test light in the retinal neural pathways that mediate color vision. Once rod signals enter a pathway, they have the same effect as the cone signals that are

Address correspondence and reprint requests to: Steven L. Buck, Department of Psychology, University of Washington, Seattle, WA 981951525, USA E-mail: sbuck@u.washington.edu also carried in that pathway. Thus, following conventional colorvision models (De Valois \& De Valois, 1993; Jameson \& Hurvich, 1955), we have suggested that rod signals combine with S-cone signals in small-bistratified ganglion-cell pathways (or other unidentified retinal S-cone pathways) and mimic the effect of S-cone signals in enhancing the amount of blue and short-wavelength red. Similarly we have suggested that rod signals combine additively with both L- and M-cone signals in midget ganglion-cell pathways but for unknown reasons have greater influence in M-cone pathways to enhance green. Because small foveal stimuli would be expected to produce little or no stimulation of rods, the localadditive explanation predicts that rod hue biases should be absent for these stimuli.

A fundamentally different remote-suppression explanation might also explain rod hue biases: Dark-adapted but unstimulated rods outside of the area of the test light might selectively modulate the strength of cone signals in the retinal pathways mediating color vision. Just such a remote-suppression mechanism (termed suppressive rod-cone interaction or SRCI) has been shown to explain the reduction of cone-mediated flicker sensitivity under darkadapted conditions (Alexander \& Fishman, 1985; Coletta \& Adams, 1984; Goldberg et al., 1983). Furthermore, Lie (1963) has shown that hue-detection thresholds rise during dark-adaptation, and Frumkes (unpublished) has suggested that the same mechanisms may alter the supra-threshold balance of hues seen in a test light. Intriguingly, Lange et al. (1997) showed that the suppression of cone flicker sensitivity by signals from dark-adapted rods surrounding a test stimulus (which he termed suppressive rod-cone interaction or SRCI) is particularly effective on small, foveal stimuli. If similar remote suppression also influences supra-threshold hue balances, then the remote-suppression explanation suggests that rod hue biases should be prominent for small stimuli presented in the central fovea. 
Thus, the two competing theoretical explanations of rod hue biases make opposite predictions about the strength of rod hue biases for small foveal stimuli: The local additive explanation predicts they should be weak or absent; the remote-suppression explanation predicts they should be strong.

The paradigm we used to reveal rod hue biases is to measure rod-mediated shifts of wavelengths of the three spectral uniquehues: unique blue, unique green and unique yellow. The wavelength of each unique hue serves as an indicator of the balance point along one perceptual opponent-hue dimensions. Thus, unique green is the blue-yellow balance point, and unique blue and unique yellow are red-green balance points. Our past work has shown that, for large extrafoveal stimuli, rods shift all three unique-hues to longer wavelengths, implying a rod red bias at unique blue, a rod blue bias at unique green, and a rod green bias at unique yellow (e.g., Buck et al., 2000; Thomas \& Buck, 2004).

\section{Materials and methods}

\section{Observers, apparatus, and stimuli}

Three observers (ages 20-25 years) having normal color vision (assessed by FM 100) participated in all conditions. Observer NH had not previously participated in psychophysical vision studies, and both observers NH and EM were naïve as to the experimental hypotheses. Each observer gave written informed consent prior to participation. All procedures and consent forms were approved by an Institutional Review Board at the University of Washington and adhered to the tenets of the Declaration of Helsinki.

The stimuli were presented with the computer-controlled Maxwellian view apparatus described in Buck (1997). The wavelength of each stimulus was varied between 420 and $630 \mathrm{~nm}$, in steps that could be as small as $1 \mathrm{~nm}$, by means of a PTR monochromator having a full bandwidth at $50 \%$ of peak transmission of $<2 \mathrm{~nm}$. Uniblitz shutters produced 1-sec presentations of each stimulus. Neutral-density filters (spectrally calibrated from 400-700 nm) controlled the illuminance of all stimuli, which was held constant at $1.5 \log$ scotopic trolands. Scotopic trolands values based on the 1951 CIE V' $(\lambda)$ standard observer (Wyszecki \& Stiles, 1982) were adjusted by the average macular pigment density at each wavelength below $535 \mathrm{~nm}$, taken from DeMarco et al. (1992). Practically, this correction influenced only the stimuli used for the measurements of unique blue and unique green.

Test stimuli were $0.2^{\circ}, 0.6^{\circ}$, or $1.0^{\circ}$ in diameter. Four tungstenspectrum pinpoint lights located $3.4^{\circ}$ above, below, left, and right of the test spot, and adjusted to be as dim as possible, guided foveation of the small, intermittently-presented test stimuli.

\section{Procedure}

A randomly interleaved double-staircase procedure was used to measure the wavelength associated with each of the three spectral unique hues. Observers were instructed to respond on a keyboard to indicate how the stimulus presented on each trial of a condition differed from their concept of a specified unique hue. For example, if asked to look for unique green, the observer was constrained to respond that each stimulus was either too yellow or too blue. Initial values of each staircase were separated by $50-70 \mathrm{~nm}$ depending on the condition and centered on the approximate expected uniquehue wavelength. Subsequent step sizes approximately halved the difference between the prior two reversals or starting points. The condition ended after each of the 2 staircases had made eight reversals. Subsequent data analyses are based on the mean of the wavelength values of the last four of these reversals for each staircase. The actual step sizes for the final reversals for the data presented here are not recoverable but in other testing typically approached closely the minimum 1-nm step size. Each staircase run determined the locus of a single unique hue. Within a staircase run, all stimuli were scotopically equated in the manner noted earlier.

\section{Adaptation conditions}

In order to measure the influence of rod signals on hue appearance, we compared unique hue judgments made to physically identical stimuli under two different adaptation conditions.

\section{Dark-adapted condition}

Before beginning an experimental session observers darkadapted (DA) the right eye for $30 \mathrm{~min}$. in order to maximize rod influence.

\section{Bleached condition}

Rod influence was minimized by making judgments from 3-8 min following exposure to a xenon flash $(0.5 \mathrm{~J}, 3.3-\mathrm{ms}$, from a Quantum Q-Flash, model T). The bleaching flash subtended $17^{\circ}$ diameter and was centered on the fovea. The minimum interval between successive bleaching flashes was $15 \mathrm{~min}$. Prior testing has confirmed that this bleaching flash is sufficient to extend the cone plateau and yield stable hue percepts for at least 8 minutes (Knight $\&$ Buck, 2002). The flash unit, energy, and bleach size used here are identical to those used in prior studies that demonstrated rod hue biases with larger, peripheral stimuli (Buck et al., 2000; Buck \& Knight, 2003; Knight \& Buck, 2002; Thomas \& Buck, 2004). However, the extremely brief duration of the flash precludes our measurement of spectral radiance and calculation of color temperature, retinal illuminance, or pigment bleaching.

Observers determined wavelengths for all three spectral unique hues for a single stimulus size under both the DA and the BL condition on the same day. The order of testing of unique hues was counterbalanced across days, to the extent possible. All adaptation conditions of one type, either DA or BL, were run together, with each type run first on half the days. Each condition using the $1.0^{\circ}$ or $0.6^{\circ}$-diameter stimulus was tested on four different days. Because of their greater difficulty and resulting variability, each condition using the $0.2^{\circ}$-diameter stimulus was tested on 8 different days.

\section{Results}

Table 1 shows the mean unique-hue wavelength settings (in $\mathrm{nm}$ ) for each stimulus and adaptation condition for each observer. Subsequent analysis and discussion makes use of a measure of rod influence on unique-hue wavelength that was calculated by subtracting the wavelength (in $\mathrm{nm}$ ) found under the BL condition from that found under the corresponding DA condition. This rod influence measure was calculated for each day's data and is reported as the mean and \pm 1 standard error of the mean of the daily differences, as shown in Table 2 and Fig. 1. Table 2 also shows the results of $t$-tests (2-sample, equal-variance) that evaluated whether the observed rod influence was $\geq 0$, performed for each condition for each observer. Because the direction of rod influence of the usual extra foveal result is a positive value (shift of unique hue to longer wavelength), a one-tailed test was performed for all rod 
Table 1. Unique hue wavelengths for all observers and conditions (mean and s.e. of daily sessions)

\begin{tabular}{|c|c|c|c|c|c|c|c|c|c|c|c|c|c|}
\hline \multirow{2}{*}{$\begin{array}{c}\text { Unique } \\
\text { Hue }\end{array}$} & \multirow{2}{*}{$\begin{array}{l}\text { Test } \\
\text { Size }\end{array}$} & \multicolumn{4}{|c|}{ ES } & \multicolumn{4}{|c|}{$\mathrm{NH}$} & \multicolumn{4}{|c|}{ LT } \\
\hline & & DA & s.e. & BL & s.e. & DA & s.e. & BL & s.e. & DA & s.e. & BL & s.e. \\
\hline \multirow[t]{3}{*}{ Yellow } & $0.2^{\circ}$ & 578.3 & 1.5 & 577.9 & 1.4 & 564.9 & 1.1 & 567.4 & 1.1 & 568.6 & 0.8 & 563.7 & 0.5 \\
\hline & $0.6^{\circ}$ & 578.1 & 2.0 & 577.2 & 1.6 & 565.3 & 1.4 & 568.3 & 1.5 & 570.2 & 0.3 & 569.3 & 2.0 \\
\hline & $1.0^{\circ}$ & 579.7 & 0.6 & 574.5 & 2.1 & 564.9 & 1.8 & 562.4 & 0.7 & 574.0 & 2.6 & 566.7 & 0.8 \\
\hline \multirow[t]{3}{*}{ Green } & $0.2^{\circ}$ & 509.4 & 5.0 & 513.8 & 4.7 & 509.3 & 3.1 & 515.0 & 2.3 & 519.2 & 3.3 & 518.4 & 4.9 \\
\hline & $0.6^{\circ}$ & 506.0 & 1.5 & 505.0 & 3.7 & 514.5 & 4.5 & 512.3 & 2.4 & 511.6 & 5.1 & 507.4 & 1.8 \\
\hline & $1.0^{\circ}$ & 511.1 & 5.5 & 506.1 & 3.1 & 531.3 & 2.4 & 513.5 & 6.6 & 496.2 & 2.7 & 496.9 & 1.7 \\
\hline \multirow[t]{3}{*}{ Blue } & $0.2^{\circ}$ & 471.2 & 3.0 & 474.2 & 2.0 & 472.1 & 1.7 & 470.1 & 1.6 & 464.9 & 2.6 & 460.6 & 2.8 \\
\hline & $0.6^{\circ}$ & 477.9 & 1.5 & 473.6 & 1.4 & 476.0 & 0.8 & 475.9 & 1.9 & 470.4 & 4.3 & 467.7 & 2.0 \\
\hline & $1.0^{\circ}$ & 471.6 & 2.1 & 474.1 & 4.3 & 477.4 & 1.9 & 475.9 & 2.1 & 469.4 & 1.4 & 469.5 & 0.6 \\
\hline
\end{tabular}

influences $\geq 0$. No values of rod influence $<0$ were found to be statistically significant for a two-tailed test. Independent of statistical significance, values of rod influence much less than $\pm 5 \mathrm{~nm}$ are of questionable generality and perceptual importance.

Fig. 1 shows the direction and magnitude of rod influence on the wavelengths of the three spectral unique-hues for the 3 test sizes $\left(0.2^{\circ}, 0.6^{\circ}\right.$, and $1^{\circ}$ diameter $)$ and three observers. For each condition, the results for observers ES, NH, and LT are shown left to right. Rod influence values above zero on the y-axis indicate that rods shift unique-hue loci to longer wavelengths, which is the direction of the rod hue biases found for large stimuli presented outside the fovea (e.g., Buck et al., 2000). Values below zero indicate that rods shift unique-hue loci to shorter wavelengths. Asterisks shows which results indicate rod hue biases that are significantly greater than zero $(P<.05)$.

For none of the three sizes of foveal test stimuli are the rod hue biases as large or consistent as those we have previously reported for extra foveal conditions (Buck et al., 2000; Buck \& Knight, 2003; Knight \& Buck, 2002; Thomas \& Buck, 2004). It is only the largest of the foveal stimuli $\left(1^{\circ}\right.$ diameter, on right in Fig. 1$)$, the one most likely to stimulate rods, which produces the largest (for unique green) and the most consistent (for unique yellow) rod hue biases. For the two smaller stimulus sizes $\left(0.2^{\circ}\right.$ and $0.6^{\circ}$ diameter $)$, the effects are generally smaller, and less consistent across observers. There were no sizable or consistent shifts of unique blue at any of the sizes tested. A more detailed analysis of these results follows.

For unique yellow (top panel), all three observers show a positive shift with the $1^{\circ}$ stimulus, with two of three observers showing statistically significant effects in the 5-10 $\mathrm{nm}$ range typically reported with extra foveal stimuli. With the $0.2^{\circ}$ and $0.6^{\circ}$ stimuli, one observer shows a small but consistent negative shift, one observer shows essentially no effect, and the remaining observer shows a normal-size positive shift for one size but not the other.

For unique green (middle panel), two of three observers show a positive shift with the $1^{\circ}$ stimulus, with only one observer showing a substantial, statistically significant $15-20 \mathrm{~nm}$ effect comparable to what is typically found for extra foveal stimuli. For the smaller stimuli, the size of shifts tends to be smaller than the error bars. However, there is a suggestion that for the smallest stimulus $\left(0.2^{\circ}\right.$, left column $)$, there is a reversal of direction toward a negative shift for two observers. Further testing would be needed to determine the validity and generality of this yellow bias, which could result from a slight chromatic adaptation by the bleaching flash.

For unique blue (bottom panel), all shifts are small for all stimulus sizes, few are as large as the error bars, only one is statistically significant, and there is no discernable trend over sizes or observers.

Table 2. Rod influence (in $\mathrm{nm}$ ): descriptive and inferential statistics

\begin{tabular}{|c|c|c|c|c|c|c|c|c|c|c|c|c|c|}
\hline \multirow{2}{*}{$\begin{array}{l}\text { Unique } \\
\text { hue }\end{array}$} & \multirow{2}{*}{$\begin{array}{l}\text { Test } \\
\text { size }\end{array}$} & \multicolumn{4}{|c|}{ ES } & \multicolumn{4}{|c|}{$\mathrm{NH}$} & \multicolumn{4}{|c|}{ LT } \\
\hline & & mean & s.e. & $\mathrm{t}$ & $P<$ & mean & s.e. & $\mathrm{t}$ & $P<$ & mean & s.e. & $\mathrm{t}$ & $P<$ \\
\hline \multirow[t]{3}{*}{ Yellow } & $0.2^{\circ}$ & 0.5 & 1.7 & 0.3 & 0.39 & -2.5 & 1.2 & -2.0 & 0.06 & 5.8 & 1.7 & 3.5 & 0.01 \\
\hline & $0.6^{\circ}$ & 0.9 & 1.5 & 0.6 & 0.29 & -3.0 & 0.8 & -3.6 & 0.01 & 0.9 & 2.1 & 0.4 & 0.33 \\
\hline & $1.0^{\circ}$ & 5.3 & 2.2 & 2.4 & 0.03 & 2.6 & 1.8 & 1.4 & 0.11 & 7.3 & 1.8 & 4.1 & 0.00 \\
\hline \multirow[t]{3}{*}{ Green } & $0.2^{\circ}$ & -4.5 & 4.8 & -0.9 & 0.36 & -5.7 & 4.0 & -1.4 & 0.18 & 0.3 & 5.4 & 0.0 & 0.48 \\
\hline & $0.6^{\circ}$ & 1.0 & 3.6 & 0.3 & 0.40 & 2.2 & 4.6 & 0.5 & 0.33 & 4.2 & 6.8 & 0.6 & 0.28 \\
\hline & $1.0^{\circ}$ & 5.1 & 3.5 & 1.4 & 0.10 & 17.8 & 6.8 & 2.6 & 0.02 & -0.7 & 3.4 & -0.2 & 0.85 \\
\hline \multirow[t]{3}{*}{ Blue } & $0.2^{\circ}$ & -3.0 & 2.9 & -1.1 & 0.31 & 2.0 & 1.7 & 1.2 & 0.13 & 3.8 & 4.2 & 0.9 & 0.20 \\
\hline & $0.6^{\circ}$ & 4.3 & 1.9 & 2.2 & 0.04 & 0.2 & 2.4 & 0.1 & 0.47 & 2.7 & 2.6 & 1.1 & 0.17 \\
\hline & $1.0^{\circ}$ & -2.5 & 3.2 & -0.8 & 0.47 & 1.5 & 3.1 & 0.5 & 0.33 & -0.1 & 1.8 & -0.1 & 0.95 \\
\hline
\end{tabular}




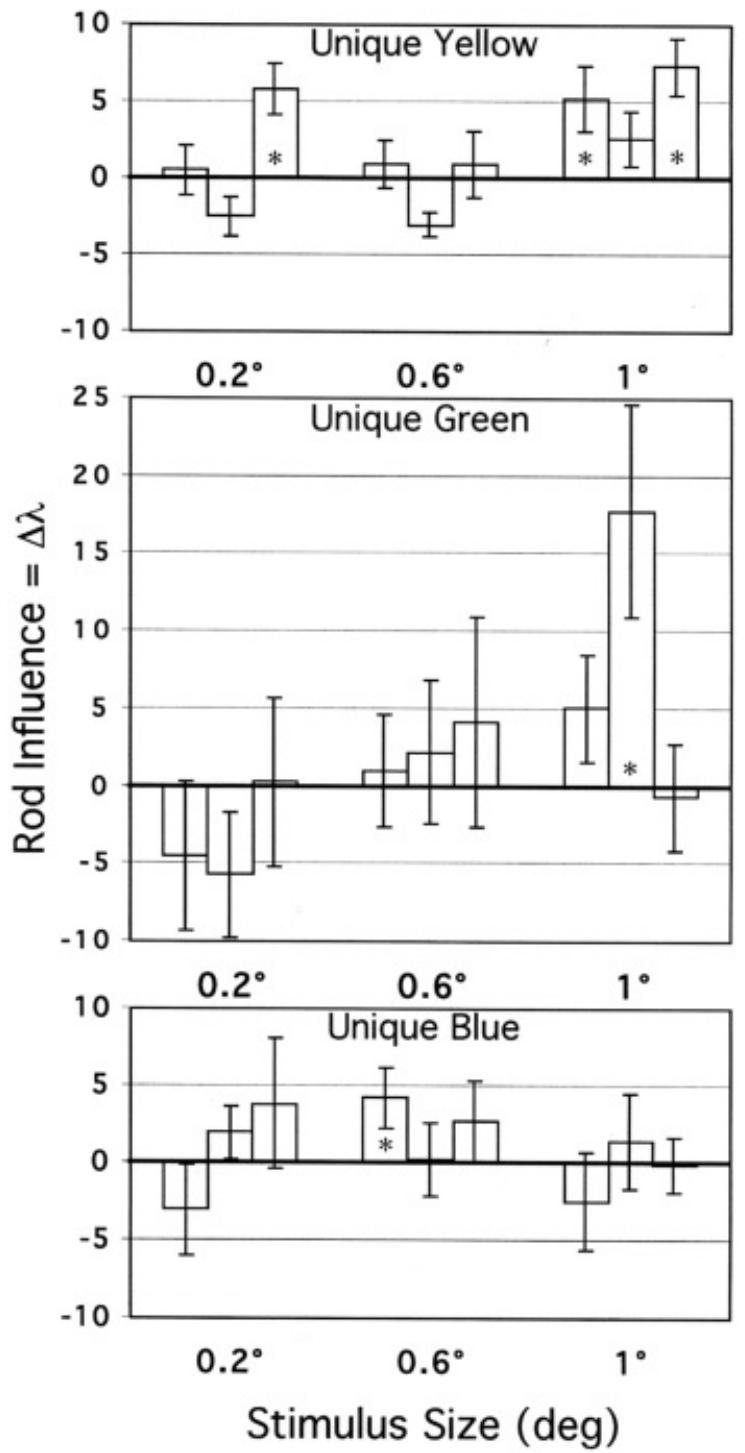

Fig. 1. The magnitude of rod influence is shown (on ordinate) by the shift, in $\mathrm{nm}$, of the wavelengths of unique yellow (top panel), unique green (middle panel), and unique blue (bottom panel) for three sizes of foveallycentered test stimuli (on abscissa). Within each condition, the data bars show results for observers ES, NH, and LT, from left to right. Rod influence values represent the mean wavelength difference between darkadapted and cone-plateau conditions for each hue/size condition. Error bars represent \pm 1 standard error of the mean of the daily differences for each condition. Asterisks indicate rod influence that is statistically $>0 \mathrm{~nm}$ $(P<.05$, one-tailed $)$.

\section{Discussion}

The pattern of rod hue biases previously reported for extra foveal stimuli (shifts to longer wavelengths of all three spectral unique hues) was not found for the smallest foveal stimuli $\left(0.2^{\circ}\right.$ and $0.6^{\circ}$ diameter), which minimize rod stimulation. Only the largest foveal stimulus ( $1^{\circ}$ diameter), the one most likely to stimulate rods, gave a suggestion of the classic extra foveal pattern of rod hue biases. These results strongly support the local additive model, which is based on the idea that rod hue biases result from the differential combination of rod and cone signals, evoked by the test stimulus, in the neural pathways that subserve color vision. The results provide no support for the remote-suppression model, which is based on the idea that rod hue biases result from the differential suppressive effects of signals from unstimulated, dark-adapted rods, located outside of the area of the test stimulus.

A substantial and long-standing literature makes clear that remote suppression provides an excellent account of rod-mediated reduction of photopic flicker sensitivity (Alexander \& Fishman, 1985; Coletta \& Adams, 1984; Goldberg et al., 1983) and that small foveal stimuli, like those used in this study, are highly susceptible to these effects on flicker sensitivity (Lange et al., 1997). However, the present study makes clear that analogous effects are not apparent for rod hue biases.

A practical implication of these results is that foveally centered stimuli as small as $1^{\circ}$ diameter are not immune from rod hue biases. These effects were generally modest (mostly shifts of unique hues of $5 \mathrm{~nm}$ or less) but a larger effect $(>15 \mathrm{~nm} \mathrm{shift})$ was found for one observer for unique green. Effects that were larger than the error bars were found for all three observers at unique yellow, for two of three observers at unique green, and for no observers at unique blue. Even for the smaller stimuli, each of the three observers showed a bleached/dark-adapted difference that exceeded the standard error of the mean in at least one condition but there was no consistent pattern across observers as to which conditions produced these differences.

Of both practical and theoretical importance, the absence of consistent differences between flash-bleached and dark-adapted conditions for the two smallest foveal stimuli suggests that the differences observed in past studies, and interpreted as rod hue biases, are not caused by bleaching-light aftereffects. On the assumption that hue judgments of both small and large stimuli would be similarly biased by any chromatic adaptation resulting from a bleaching light, the present findings eliminate a potential confound to the conclusion that rod signals shift hue balances.

\section{References}

Alexander, K.R. \& Fishman, G.A. (1985). Rod-cone interaction in flicker perimetry: Evidence for a distal retinal locus. Documenta Ophthalmologica 60, 3-36.

BucK, S. (1997). Influence of rod signals on hue perception: Evidence from successive scotopic color contrast. Vision Research 37, 1295-1301.

Buck, S. \& KNight, R. (2003). Stimulus duration affects rod influence on hue perception. In Normal and Defective Colour Vision, eds. Mollon, J.D., Pokorny, J. \& Knoblauch K., pp. 177-184. Oxford: Oxford University Press.

Buck, S., Knight, R. \& Bechtold, J. (2000). Opponent-color models and the influence of rod signals on the loci of unique hues. Vision Research 40, 3333-3344.

Buck, S., Knight, R., Fowler, G. \& Hunt, B. (1998). Rod influence on hue-scaling functions. Vision Research 38, 3259-3263.

Coletta, N.J. \& AdAMS, A.J. (1984). Rod-cone interaction in flicker detection. Vision Research 24, 1333-1340.

DeMarco, P., Pokorny, J. \& Smith, V. (1992). Full-spectrum cone sensitivity functions for X-chromosome-linked anomalous trichromats. Journal of the Optical Society of America A 9, 1465-1476.

De Valois, R.L. \& De Valois, K.K. (1993). A multi-stage color model. Vision Research 33, 1053-1065.

FRUMKES, T. (unpublished). Dark-adapted rods suppress color opponency. Presentation made at the XIVth Symposium of the International Research Group on Colour Vision Deficiencies. Ghent, 1997.

Goldberg, S.H., Frumkes, T.E. \& NygaARd, R.W. (1983). Inhibitory influence of unstimulated rods in the human retina: Evidence provided by examining cone flicker. Science 221, 180-182. 
JAmeson, D. \& Hurvich, L. (1955). Some quantitative aspects of an opponent-colors theory. I. Chromatic responses and spectral saturation. Journal of the Optical Society of America 45, 546-552.

Knight, R. \& BucK, S. (2002). Time-dependent changes of rod influence on hue perception. Vision Research 42, 1651-1662.

Lange, G., Denny, N. \& Frumkes, T.E. (1997). Suppressive rod-cone interactions: Evidence for separate retinal (temporal) and extraretinal (spatial) mechanisms in achromatic vision. Journal of the Optical Society of America 14, 2487-2498.
LIE, I. (1963). Dark adaptation and the photochromatic interval. Documenta Ophthalmologica 17, 411-510.

Thomas, L. \& Buck, S. (2004). Generality of Rod Hue Biases with Smaller, Brighter, and Photopically Specified Stimuli. Visual Neuroscience 21, 257-262.

Wyszecki, G. \& StILEs, W. (1982). Color science: Concepts and methods, quantitative data and formulae, 2nd edition. New York: Wiley. 